\title{
THE INTENSITY OF SHORT LIGHT PULSES DETERMINED WITH SATURABLE ABSORBERS
}

\author{
A. PENZKOFER, D. VON DER LINDE and A. LAUBEREAU \\ Physik-Department der Technischen Universitat München, Munich, Germany
}

Received 15 November 1971

\begin{abstract}
It is shown that the peak intensity of a short light pulse can be obtained from a measurement of the energy transmission using a saturable absorber. The influence of temporal and spatial pulse shape is discussed and found to be of minor importance. The method is independent of the beam diameter No absolute calibration of the photodetectors is necessary. The technique is demonstrated experimentally, and good agreement is found with calculations.
\end{abstract}

In general, the intensity of picosecond light pulses is determined from the pulse energy, the pulse duration and the beam diameter. These three parameters have to be measured simultaneously to estimate the peak intensity $I_{O}$ of the light pulses. For a more accurate determination of $I_{0}$ the pulse shape and intensity profile across the beam must be known.

In this letter we wish to show that the peak intensity of a short pulse may be obtained from a measurement of the fraction of energy transmitted by a saturable dye (energy transmission $T_{\mathrm{E}}$ ). It will be discussed that this method is independent of the beam diameter and depends only slightly on pulse duration, temporal pulse shape and spatial beam profile. Once the relationship between pulse intensity and energy transmission has been established, the measured ratio of transmitted to incident pulse energy $T_{\mathrm{E}}=E_{\mathrm{tr}} / E_{\mathrm{i}}$ gives directly the peak pulse intensity $I_{\mathrm{O}^{\prime}}$ The incident energy is given by:

$E_{\mathrm{i}}=2 \pi(\Delta r)^{2} \Delta t I_{\mathrm{o}} \int s\left(t^{\prime}, r^{\prime}\right) r^{\prime} \mathrm{d} r^{\prime} \mathrm{d} t^{\prime}$,

where the function $s\left(t^{\prime}, r^{\prime}\right)=s(t / \Delta t, r / \Delta r)$ accounts for the pulse shape and the transverse intensity distribution. $\Delta t$ and $\Delta r$ are the pulse duration (fwhm) and the beam radius (hwhm), respectively. Assuming that the intensity varies slowly with distars, $r$ from the beam axis (as compared to the optical wavelength) the transmitted energy may be written:

$$
\begin{aligned}
E_{\mathrm{tr}} & =2 \pi(\Delta r)^{2} \Delta t I_{\mathrm{o}} \\
& \times \int s\left(t^{\prime}, r^{\prime}\right) T\left(T_{\mathrm{O}}, I_{\mathrm{o}} s\left(t^{\prime}, r^{\prime}\right), \tau / \Delta t\right) r^{\prime} \mathrm{d} r^{\prime} \mathrm{d} t^{\prime} .
\end{aligned}
$$

$\tau$ denotes the recovery time of the bleachable dye.

Using eqs. (1) and (2) we obtain the energy transmission

$T_{\mathrm{E}}=K^{-1} \int s\left(t^{\prime}, r^{\prime}\right) T\left(T_{\mathrm{O}}, I_{\mathrm{O}} s\left(t^{\prime}, r^{\prime}\right), \tau / \Delta t\right) r^{\prime} \mathrm{d} r^{\prime} \mathrm{d} t^{\prime}$

The constant $K$ is given by $K=\int s\left(t^{\prime}, r^{\prime}\right) r^{\prime} \mathrm{d} r^{\prime} \mathrm{d} t^{\prime}$. It is seen from eq. (3) that the energy transmission depends on the initial transmission $T_{\mathrm{O}}$, the peak intensity $I_{0}$, the shape function $s$ and the ratio $\tau / \Delta t$. It should be noted that the beam radius $\Delta r$ does not enter eq. (3). The transient transmission factor $T$ of the saturable dye on the right side of eq. (3) is obtained from the integral cquation,

$$
\begin{aligned}
& T\left(T_{\mathrm{O}}, I_{\mathrm{O}} s\left(t^{\prime}, r^{\prime}\right), \tau / \Delta t\right)=T_{\mathrm{O}} \exp \left\{\frac{2 \sigma}{h \nu} I_{\mathrm{O}} \int_{-\infty}^{t^{\prime}} s\left(l^{\prime \prime}, r^{\prime}\right)\right. \\
& \left.\times\left[1-T\left(T_{\mathrm{O}}, I_{\mathrm{O}} s^{\prime \prime}\left(t^{\prime \prime}, r^{\prime}\right), \tau / \Delta t\right)\right] \exp \left\{\left(t^{\prime \prime}-t^{\prime}\right) / \tau\right\} \mathrm{d} t^{\prime \prime}\right\} 。
\end{aligned}
$$

( $\sigma$ absorption cross section, $\nu$ light frequency), which has been derived for a two-level system [1].

Eqs. (3) and (4) state a one-to-one correspondence between energy transmission $T_{\mathrm{E}}$ and the peak intensity $I_{O}$ for a given initial transmission. The dependence on pulse shape, beam profile and the ratio $\tau / \Delta l$ will be discussed below. This relationship allows to infer the peak intensity $I_{0}$ of a short light pulse from the measurement of the energy transmission $T_{\mathrm{E}}$. Now, we wish to show that a good estimate of the peak intensity is obtained without the use of calibrated detectors and without a detailed knowledge of pulse duration, pulse shape and beam profile. 


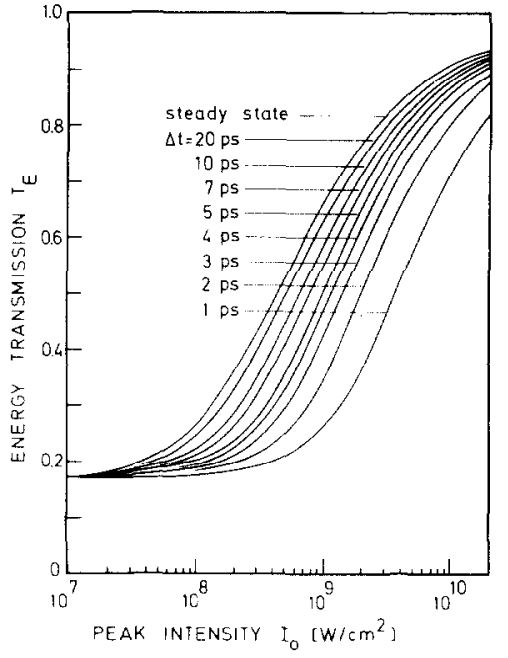

Fig. 1. Calculated energy transmission $T_{\mathrm{E}}$ as a function of peak intensity $I_{0}$ for several values of the pulse duration $\Delta l$ : dye parameters: $T_{0}=0.173, \sigma=1.34 \mathrm{x}$ $\times 10^{-16} \mathrm{~cm}^{2}, \tau-9.1 \mathrm{psec}$. corresponding to the dye of ref. [2]. Gaussian temporal and spatial pulse shape.

Firstly, the influence of the pulse duration will be examined. Solving eqs. (3) and (4) numerically the energy transmission $T_{\mathrm{E}}$ is calculated for various values of $\Delta t$. Fig. 1 shows the set of curves obtained for pulses with temporal and spatial gaussian pulse shapes (TEM 00 -mode) and for the dye parameters $\sigma-1.84 \times 10^{-16} \mathrm{~cm}^{2}$, $\tau=9.1$ psec [2]. The calculated energy transmission is plotted as a function of the incident peak intensity $I_{O}$.

In the steady-state situation $[3](\tau / \Delta t \ll 1)$ the energy transmission is independent of the pulse duration. The transmission curve for $\Delta t=20 \mathrm{psec}$ $(\tau / \Delta l=0.455)$ differs only slightly from the steady-state curve. It is easily seen from fig. 1 that for pulses of duration $\Delta l \geqslant 20 \mathrm{psec}$ the influence of $\Delta t$ on $T_{\mathrm{E}}$ may be neglected. For shorter pulses the curves deviate increasingly from the steady-state curve. If we allow $\Delta t$ to vary between 4 and 10 psec the corresponding value of $I_{0}$ changes approximately by a factor of 1.7 (at $T_{\mathrm{E}}=0.5$ ).

Secondly, we consider different transverse intensity distributions of the laser beam. Fig. 2a shows the transmission $T_{\mathrm{E}}$ calculated for a rectangular beam profile, a $\mathrm{TEM}_{00}$-mode (gaussian profile), a TEM 01 -mode (for circular shaped mirrors), and for the diffraction pattern of a circular aperture. The time dependence of the pulse is assumed to be gaussian in all cases. Fig. $2 \mathrm{a}$
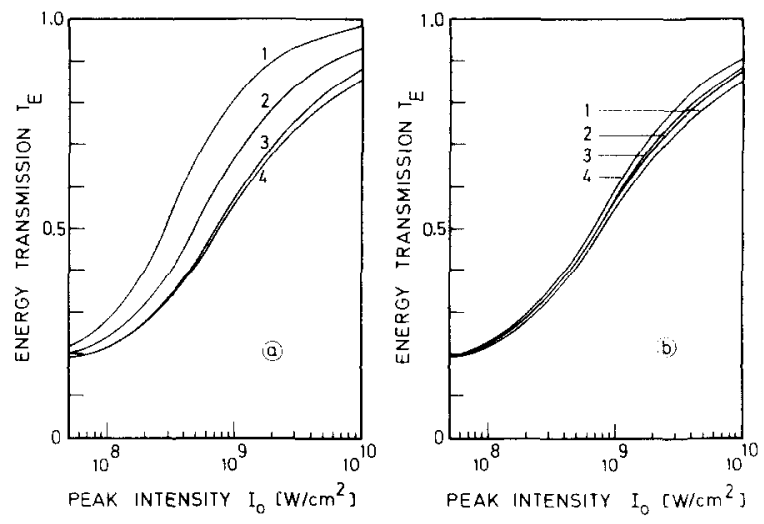

Fig. 2. (a) Energy transmission $T_{E}$ versus peak intensity $I_{0}$ for several transverse intensity distributions: (1) rectangular beam profile, (2) $\mathrm{T} E \mathrm{M}_{00}$-mode, (3) TEMi $\mathrm{H}_{01}$-mode, (4) diffraction pattern of a circular aperture. Gaussian temporal pulse shape. (b) Energy transmission $T_{E}$ versus peak intensity $I_{O}$ for different temporal pulse shapes: (1) lorentzian. (2) hyperbolic secant, (3) gaussian, (4) rectangular pulse. Gaussian beam profile. In fig. (a) and (b) pulses of 8 psec duration (fwhm) and the parameter values of the dye of ref. [2] have been assumed. $T_{0}=0.173$.

demonstrates that the transmission curves do not differ strongly. In particular, the deviations are small for the profiles of physical interest: the $\mathrm{TEM}_{00}$, the TEM $\mathrm{M}_{01}$ and the diffraction pattern. For example, at $T_{\mathrm{E}}=0.5$ we calculate $I_{\mathrm{O}}$ to be $5 \times 10^{8}$ and $7 \times 10^{8} \mathrm{~W} / \mathrm{cm}^{2}$ for $\mathrm{TEM}_{00}$ and TEMO1-mode patteris, respectively.

Finally, the effect of the detailed time dependence of the pulse on the energy transmission is investigated. Fig. $2 \mathrm{~b}$ shows transmission curves calculated for a rectangular, gaussian, hyperbolic secant, and lorentzian pulse shape. A spatial gaussian beam profile was assumed in the calculations. It is seen from fig. $2 \mathrm{~b}$ that the influence of the pulse shape is very small. At $T_{\mathrm{E}}$ $=0.5$ we find intensity values of $6.7 \times 10^{8}$ to $8.0 \times 10^{8} \mathrm{~W} / \mathrm{cm}^{2}$ for the various pulse shapes. The peak intensity values corresponding to gaussian and hyperbolic secant pulses deviate by a few per cent, only.

The energy transmission of the dye of ref. [2] was investigated experimentally. A mode-locked Nd:glass laser was used which generated pulses of known duration and shape [4]. The intensity distribution of the laser beam was carefully determined. It was found that the beam profile, to a good approximation, corresponded to a TEM $\mathrm{M}_{00}$ mode (gaussian beam profile). A calibrated photodiode maasured the pulse energy. Background energy content was negligible in our experiments 


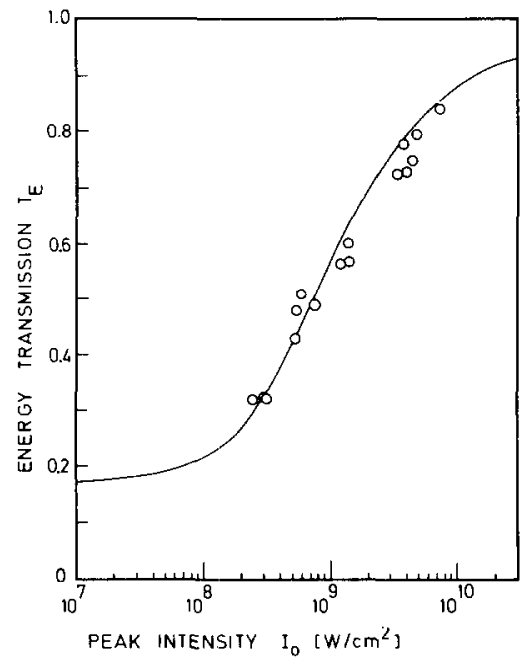

Fig. 3. Comparison of the measured and calculated energy transmission (initial transmission $T_{0}=0.173$ ). The curve was calculated using the data of ref, [2] and pulse parameters corresponding to the experimental light pulses ( 8 psec duration, approximately gaussian temporal and spatial pulse shape).

[5]. Knowing the pulse energy, the duration $(\Delta t=8 \mathrm{psec})$, the shape (approximately gaussian), and the beam profile, we are in the position to calculate the peak intensity $I_{\mathrm{o}}$ and to compare it with the measured energy transmission of the saturable dye.

In fig. 3 the energy transmission $T_{E}$ is plotted as a function of the experimental value of $I_{0}$. The initial transmission of the dye was adjusted to $T_{0}=0.173$ (cell length $5 \mathrm{~mm}$ ). The solid line in fig. 3 was calculated using the published data of the dye [2] and the characteristic data of our picosecond pulses. The curve accounts well for the experimental points. The good agreement be- tween calculation and experiment supports the two-level model for the dye molecule. It should be noted that for pulse intensities larger than $1010 \mathrm{~W} / \mathrm{cm}^{2}$ a decrease of the transmission was observed, which indicates the onset of competing nonlinear effects of the solvent dichloroethane.

The curve of fig. 3 can be considered as a calibration curve which allows the determination of pulse intensities using measured energy transmission values. Fig. 3, which directly applies to pulses with gaussian beam profile and gaussian shape of 8 psec duration, can be used in more general cases on account of the arguments discussed above. If temporal pulse shape, spatial beam profile and pulse duration are not known, the accuracy of the intensity measurement is estimated to be a factor of 2 to 4 , provided the pulses are not shorter than $\approx 2$ psec.

The method can be used for intensity values of $10^{8}$ to $1010 \mathrm{~W} / \mathrm{cm}^{2}$. With proper optical systems (telescopes) high power laser beams are readily adjusted to these intensities.

We believe that this technique offers a simple tool for controlling the intensity of short light pulses.

The authors are indebted to Professor $\mathrm{W}$. Kaiser for many stimulating discussions.

\section{REFERENCES}

[1] J. D. Macomber, J. Appl. Phys. 38 (196ri) 3525.

[2] Eastman Kodak dye No. A 9860, data release.

[3] M. Hercher, Appl. Opt. 6 (1967) 947.

[4] D. von der Linde, O. Bernecker and W. Kaiser, Opt. Commun. 2 (1970) 149;

D. von der Linde and A. Laubereau, Opt. Commun. 3 (1971) 279.

[5] D. von der Linde, IEEE J. Quantum. Electron., to be published. 